\title{
Retracted: Multiplication of Fast Neutrons Source Flux by Using Deuterium-Helium-3 Plasma
}

\author{
ISRN High Energy Physics
}

Received 4 December 2013; Accepted 4 December 2013; Published 22 January 2014

Copyright (C) 2014 ISRN High Energy Physics. This is an open access article distributed under the Creative Commons Attribution License, which permits unrestricted use, distribution, and reproduction in any medium, provided the original work is properly cited.

The article titled "Multiplication of Fast Neutrons Source Flux by Using Deuterium-Helium-3 Plasma" [1], published in ISRN High Energy Physics, has been retracted as it is found to contain a substantial amount of material from the paper "Developmental study of a plasma source of $14 \mathrm{MeV}$ neutrons. I. General concept," V. T. Voronchev, V. I. Kukulin, B. M. Kuzhevskij, Nuclear Instruments and Methods in Physics Research Section A, vol. 525, no. 3, pp. 626-632, 2004.

\section{References}

[1] M. Mahdavi and M. Shahbahrami, "Multiplication of fast neutrons source flux by using deuterium-helium-3 plasma," ISRN High Energy Physics, vol. 2013, Article ID 689739, 4 pages, 2013. 


\author{
Received 10 April 2013; Accepted 7 May 2013 \\ Academic Editors: K. Cho and I. V. Vancea
}

Mohammad Mahdavi and Maryam Shahbahrami

Physics Department, Mazandaran University, P.O. Box 47415-416, Babolsar, Iran

Correspondence should be addressed to Mohammad Mahdavi; m.mahdavi@umz.ac.ir

Copyright ( 2013 M. Mahdavi and M. Shahbahrami. This is an open access article distributed under the Creative Commons Attribution License, which permits unrestricted use, distribution, and reproduction in any medium, provided the original work is properly cited.

The production of fast neutrons source is examined by using a thermal neutron flux inside $\mathrm{D} /{ }^{3} \mathrm{He}$ plasma. In order to reach a favorable yield of fast neutrons flux, the parameters such as energy loss rate, reaction probability, and neutron absorption length are calculated. The nuclear conversion efficiency, $\zeta$, of thermal neutron to fast neutrons is obtained to be $2 \times 10^{-3}$ by calculating the physical parameters for the $\mathrm{D} /{ }^{3}$ He plasma designed.

\section{Introduction}

With considering the importance of widespread application of neutrons' flux in various branches of science and technology, numerous efforts have always been undertaken to improve the efficiency of neutrons' sources. A special attention in this field is now focused on developing fast neutron sources. Unlike neutron beams with less energy, the choice of sufficient tools for the production of fast neutrons is restricted. In fact, the most natural way of producing an appreciable source of these neutrons is based on employing the $\mathrm{D}(\mathrm{t}, \mathrm{n})^{4} \mathrm{He}$ nuclear reaction. Different concepts of $\mathrm{D} / \mathrm{T}$ neutron sources have been proposed. However, in spite of differences in design suggested for fast neutron sources, all of them have a common feature; that is, radioactive tritium must be initially in the ion beams or in the targets. In fact, operation with tritium materials is known to be prohibited in many research centers. Thus, a developmental study of fast $(14.1 \mathrm{MeV})$ neutrons is still urgent. Some studies have been performed in this field such as production of fast neutrons by using thermal neutrons with the help of the $\mathrm{LiD}$ combination [1]. One of these applicable methods that was examined in this paper is the production of fast neutron sources in the D/ ${ }^{3}$ He plasma using a thermal neutrons flux. Avoiding the implementation of initial tritium due to its radioactive property is one of the main factors in using of
D/ ${ }^{3}$ He plasma. To provide such conditions one should use deuterium-containing materials. Secondary tritium particles are generated from the interaction of thermal neutron with the helium nucleus, ${ }^{3} \mathrm{He}(\mathrm{n}, \mathrm{p}) \mathrm{T}$ in $\mathrm{D} /{ }^{3} \mathrm{He}$ plasma. Tritium particles fuse with target deuterons and release fast neutrons 14.1 MeV as follows:

$$
\mathrm{D}+\mathrm{T} \longrightarrow{ }^{4} \mathrm{He}(3.5 \mathrm{MeV})+\mathrm{n}(14.1 \mathrm{MeV})
$$

In this work, we have examined the theory of the fast neutrons' sources production using the $\mathrm{D} /{ }^{3} \mathrm{He}$ plasma. The result of these calculations led to the attainment of the most desirable efficiency for fast neutrons flux.

\section{Theory}

In a $\mathrm{D} /{ }^{3} \mathrm{He}$ mixture irradiated by a flux of thermal neutrons, the nuclei interaction with thermal neutrons leads to three nuclear reactions:

$$
\begin{gathered}
\mathrm{n}+{ }^{3} \mathrm{He} \longrightarrow \mathrm{T}+\mathrm{p}+763 \mathrm{KeV} \\
\mathrm{n}+{ }^{3} \mathrm{He} \longrightarrow \gamma+{ }^{4} \mathrm{He}, \\
\mathrm{n}+\mathrm{D} \longrightarrow \mathrm{T}+\gamma+6.26 \mathrm{MeV}
\end{gathered}
$$


Among these three processes, the ${ }^{3} \mathrm{He}(\mathrm{n}, \mathrm{p}) \mathrm{T}$ reaction fully dominates because of a very large cross-section (see Figure 1) [2].

At the first step, tritium ions are produced from interaction of thermal neutron with helium nuclei:

$$
\mathrm{n}_{\mathrm{th}}+{ }^{3} \mathrm{He} \longrightarrow \mathrm{T}+\mathrm{p}+763 \mathrm{KeV} \text {. }
$$

The contribution of tritium and proton particles energies according to energy and momentum conservation is $191 \mathrm{KeV}$ and $572 \mathrm{KeV}$, respectively. At the second step, tritium ions fuse with plasma deuterons as follows:

$$
\mathrm{T}+\mathrm{D} \longrightarrow \mathrm{n}+\alpha+17.6 \mathrm{MeV} .
$$

The image of thermal neutrons interaction with $\mathrm{D} /{ }^{3} \mathrm{He}$ plasma that leads to production of fast neutrons is shown in Figure 2.

There are two important factors of the reaction ${ }^{3} \mathrm{He}(\mathrm{n}, \mathrm{p}) \mathrm{T}$. Firstly, this reaction contains a very large cross- section of about 5300b [3]. Secondly, the initial energy of the tritium particles produced, $E_{\mathrm{T}}^{0}=191 \mathrm{KeV}$, is very close to resonance energy, $E_{\mathrm{T}}^{\mathrm{res}}=160 \mathrm{KeV}$, of the $\mathrm{D}(\mathrm{t}, \mathrm{n})^{4} \mathrm{He}$ reaction [4]. The nuclear conversion efficiency, $\xi$, of thermal neutrons to fast neutrons can be described according to

$$
\xi=F_{\mathrm{n}^{3} \mathrm{He}} \times F_{\mathrm{TD}},
$$

where $F_{\mathrm{n}^{3} \mathrm{He}}$ is the thermal neutron absorption probability by helium nuclei and $F_{\mathrm{TD}}$ is the probability of tritium interaction with deuterons plasma. The amount of $F_{\mathrm{TD}}$ can be defined by analyzing the slowing-down of the $191 \mathrm{keV}$ tritium in the $\mathrm{D} /{ }^{3} \mathrm{He}$ plasma. Principally, fast charged particles slow down in plasma through Coulomb and nuclear elastic scattering caused by plasma electrons and ions. The contribution of (4) is negligible since the initial tritium kinetic energy is $191 \mathrm{KeV}$. Tritium Coulomb slow-down in the $\mathrm{D} /{ }^{3} \mathrm{He}$ plasma can be calculated according to the conventional binary-collision model [5]. According to the above, the average instantaneous rate of tritium energy loss, $\left\langle d E_{\mathrm{T}} / d t\right\rangle$, in Maxwellian $\mathrm{D} /{ }^{3} \mathrm{He}$ plasma can be represented as

$$
\left\langle\frac{d E_{\mathrm{T}}}{d t}\right\rangle=\sum_{j}\left\langle\frac{d E_{\mathrm{T}}}{d t}\right\rangle_{j},
$$

where the term $\left\langle d E_{\mathrm{T}} / d t\right\rangle_{j}$ defines tritium energy loss in $T-j$ scattering $\left(j=e, \mathrm{D},{ }^{3} \mathrm{He}\right)$. In order to calculate tritium energy loss, we should estimate the average rate of the particles energy loss. With the assumption that the ion of mass $M$ and charge $Z e$ with velocity $v$ in the laboratory system collides with a field particle of mass $m$ and charge $z e$ with velocity $w$, the cross-section for scattering through an angle $\theta$ in the c.m. system is;

$$
\begin{aligned}
\sigma(\theta)= & \left(\frac{Z z e^{2}}{(1 / 2)[M m / M+m](v-w)^{2}}\right)^{2} \\
& \times \frac{1}{\left[4 \sin ^{2}(\theta / 2)\right]^{2}} .
\end{aligned}
$$

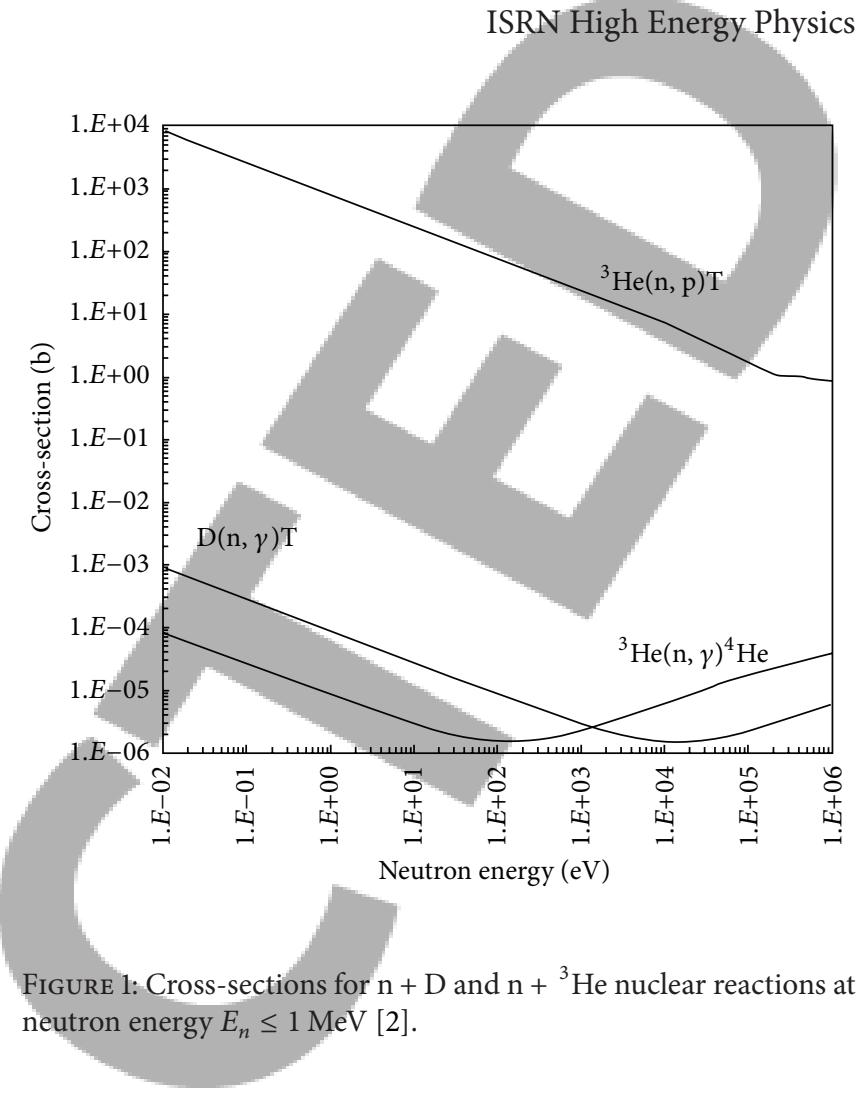

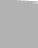

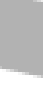

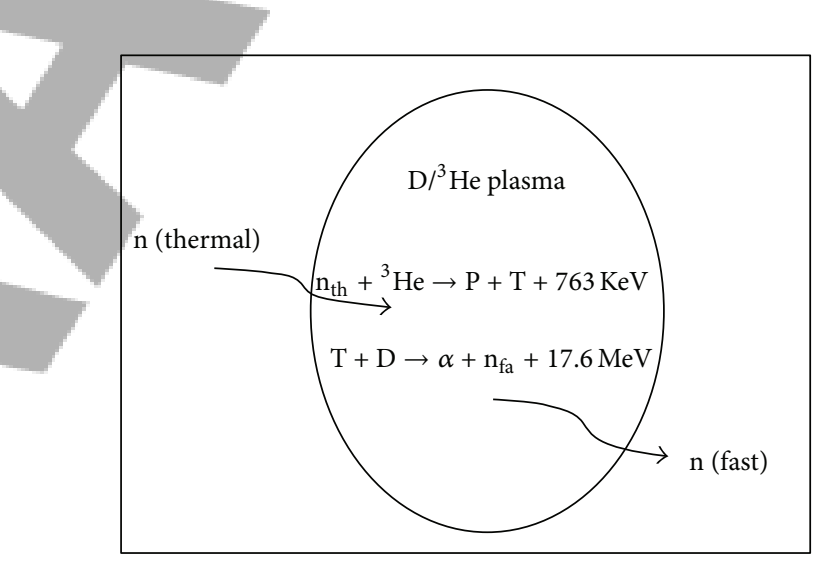

FIGURE 2: Image of collision of thermal neutron and helium nuclei in $\mathrm{D} /{ }^{3}$ He plasma.

$[m / M+m](v-w)$ is the velocity of the ion in the c.m. system. In the laboratory system, the change $\Delta E$ of the kinetic energy of the ion is [6]

$$
\begin{aligned}
\Delta E= & -\frac{m M}{(M+m)^{2}} \\
& \times\left\{2 \sin ^{2}\left(\frac{\theta}{2}\right)[(v-w)(M v+m w)]\right. \\
& \quad+\sin \theta \cos \phi|(v-w) \times(M v+m w)|\} ;
\end{aligned}
$$


here $\theta$ is the angle of scattering in the c.m. system and $\phi$ is the azimuthal angle of scattering in the c.m. system. The average value of $\sin ^{2}(\theta / 2)$ for such a collision is obtained from (7)

$$
\begin{aligned}
& \left(\sin ^{2}\left(\frac{\theta}{2}\right)\right)_{a v} \\
& \quad=\frac{\pi}{2 \sigma_{t}}\left(\frac{Z z e^{2}}{(1 / 2)[M m /(M+m)](v-w)^{2}}\right)^{2} \ln \Lambda,
\end{aligned}
$$

where $\sigma_{t}$ is the total scattering cross-section and the second term of (8) averages to zero. Thus, the average energy change of the particle from such a collision is simply

$$
\begin{aligned}
\Delta E= & -\frac{4 \pi}{\sigma_{t}} \frac{\left(Z z e^{2}\right)^{2}}{m} \times \ln \Lambda \\
& \times\left(\frac{(v-w)[v(1+(m / M))-(m / M)(v-w)]}{(v-w)^{4}}\right) .
\end{aligned}
$$

The velocity distribution of the field particles under consideration is $f(w) d w$ per unit volume. The number of collisions with field particles having velocities between $w$ and $w+d w$ in a range of time, $\delta t$, is $\sigma_{t}|v-w| f(w) d w \delta t$. Thus, the rate of change of energy of the ion determined by plasma particles with velocity $w$ is

$$
\begin{aligned}
\left(\frac{d E}{d t}\right)= & -\frac{4 \pi}{m}\left(Z z e^{2}\right)^{2} \times \ln \Lambda \\
& \times\left[\frac{(v-w)[v(1+(m / M))-(m / M)(v-w)]}{(v-w)^{3}}\right] \\
& \times f(w) d w .
\end{aligned}
$$

If we take a Maxwellian distribution,

$$
f(w)=f_{0} \exp \left[-\left(\frac{w}{w_{t}}\right)^{2}\right],
$$

where $w_{t}=\left(2 k_{B} T_{j} / m\right)^{2}$ is the thermal speed of the plasma particles. $k_{B}$ and $T_{j}$ are Boltzmann constant and temperature particles (energy), respectively. After solving the above integral, we obtain [7]

$$
\begin{gathered}
\left\langle\frac{d E_{\mathrm{T}}}{d t}\right\rangle_{j}=-\frac{4 \pi e^{4}}{\left(2 m_{j} T_{j}\right)^{1 / 2}}\left(Z_{T} Z_{j}\right)^{2} n_{j} \ln \Lambda_{j} \frac{\operatorname{Phi}\left(\zeta_{j}\right)}{\zeta_{j}} \\
\Phi\left(\zeta_{j}\right)=\operatorname{erf}\left(\zeta_{j}\right)-\frac{2}{\pi^{1 / 2}}\left(1+\frac{m_{j}}{m_{T}}\right) \zeta_{j} \exp \left(-\zeta_{j}^{2}\right), \\
\zeta_{j}=\left(\frac{m_{j} E_{\mathrm{T}}}{m_{T} T_{j}}\right)^{1 / 2},
\end{gathered}
$$

where $n_{j}$ is the density of plasma particles species $j$ having Maxwellian temperature $T_{j}$. It is assumed that $T_{\mathrm{D}}=T_{3_{\mathrm{He}}}=$ $T_{i}$ for the homogenous temperature plasma. The Coulomb logarithm in classical, and quantum mechanical limits are defined as [5]

$$
\begin{aligned}
\ln \Lambda_{j}^{\text {classical }} & =\ln \left(1+\frac{D^{2}}{\rho_{j}^{2}}\right)^{1 / 2}, \\
\ln \Lambda_{j}^{\text {quantum }} & =\ln \frac{4 \pi D}{\lambda_{j}}-\frac{1}{2},
\end{aligned}
$$

where $D$ is the Debye length and $\rho_{j}$ and $\lambda_{j}$ are the cutoff impact parameter and the scattering wave length in the $(T-j)$ system, respectively. During the slowing down of the $191 \mathrm{KeV}$ tritium particle, its kinetic energy transfers to plasma electrons and ions. The fraction of energy $\eta_{j}$ transferred to plasma species $j$ is introduced as follows:

$$
\eta_{j}=\frac{1}{E_{\mathrm{T}}^{0}-E_{\mathrm{th}}} \int_{E_{\mathrm{th}}}^{E_{\mathrm{T}}^{0}}\left(\frac{\left\langle d E_{\mathrm{T}} / d t\right\rangle_{j}}{\left\langle d E_{\mathrm{T}} / d t\right\rangle}\right) d E_{\mathrm{T}},
$$

where $E_{\mathrm{th}}$ is the ion thermal energy, $(3 / 2) k_{B} T_{i}$. The fractions of energy transferred to electrons, nuclei ${ }^{3} \mathrm{He}$ and $\mathrm{D}$ are calculated using (15) as $0.6,0.3$, and 0.1 , respectively. Therefore, tritium energy is mainly deposited to the plasma electrons. $F_{\mathrm{TD}}$ is an important parameter that is effective in nuclear conversion efficiency calculation and is defined as fusion probability of slowed-down tritium ions and background deuterons of $\mathrm{D} /{ }^{3} \mathrm{He}$ plasma. The differential reaction probability, $d F_{\mathrm{TD}}\left(E_{\mathrm{T}}\right)$, at tritium energy, $E_{\mathrm{T}}$, is given in the following form [8]:

$$
d F_{\mathrm{TD}}\left(E_{\mathrm{T}}\right)=\left(1-F_{\mathrm{TD}}\left(E_{\mathrm{T}}\right)\right) \frac{n_{D} \sigma\left(E_{\mathrm{T}}\right) d E_{\mathrm{T}}}{\left\langle d E_{\mathrm{T}} / d x\right\rangle} d E_{\mathrm{T}},
$$

(11) where $\sigma\left(E_{\mathrm{T}}\right)$ is the $\mathrm{D}$-T reaction cross-section. The probability, $F_{\mathrm{TD}}(E)$, of reaction during tritium slowing from the initial energy, $E_{\mathrm{T}}^{0}$, to an energy $E$ equals:

$$
F_{\mathrm{TD}}(E)=1-\exp \times\left(\int_{E}^{E_{\mathrm{T}}^{0}}\left(\frac{2 E_{\mathrm{T}}}{m_{\mathrm{T}}}\right)^{1 / 2} \frac{n_{D} \sigma\left(E_{\mathrm{T}}\right)}{\left\langle d E_{\mathrm{T}} / d t\right\rangle} d E_{\mathrm{T}}\right) .
$$

\section{Conclusion}

The probability $F_{\mathrm{TD}}\left(E_{\mathrm{th}}\right)$ of the $\mathrm{D}(\mathrm{t}, \mathrm{n}){ }^{4} \mathrm{He}$ reaction, when tritium decreases to thermal energy, $E_{\mathrm{th}}$, is illustrated in Figure 3. As it is seen, the value of $F_{\mathrm{TD}}\left(E_{\mathrm{th}}\right)$ rises with increasing $T_{e}$. Also, the change of $D /{ }^{3} \mathrm{He}$ plasma density does not significantly affect the value of $F_{\mathrm{TD}}\left(E_{\mathrm{th}}\right)$. Figure 4 shows the reaction probability in terms of plasma ion density $\mathrm{n}_{i}=$ $\mathrm{n}_{\mathrm{D}}+\mathrm{n}_{3_{\mathrm{He}}}$ in the $10^{19}-10^{23} \mathrm{~cm}^{-3}$ range. Another parameter which can determine the size of the plasma is the length of neutron absorption (mean free path) that is indicant of an average distance traversed by the neutron for the creation of first collision. If the generator system does not include a neutron reflector, the plasma size should be comparable with the length of neutron absorption, $L_{\mathrm{n}}$ via the ${ }^{3} \mathrm{He}(\mathrm{n}, \mathrm{p}) \mathrm{T}$ reaction. The absorption length is $L_{\mathrm{n}}=\left(\mathrm{n}_{3_{\mathrm{He}}} \bar{\sigma}_{\mathrm{np}}\right)^{-1}$ which is the ${ }^{3} \mathrm{He}(\mathrm{n}, \mathrm{p})$ reaction cross-section for the most probable 


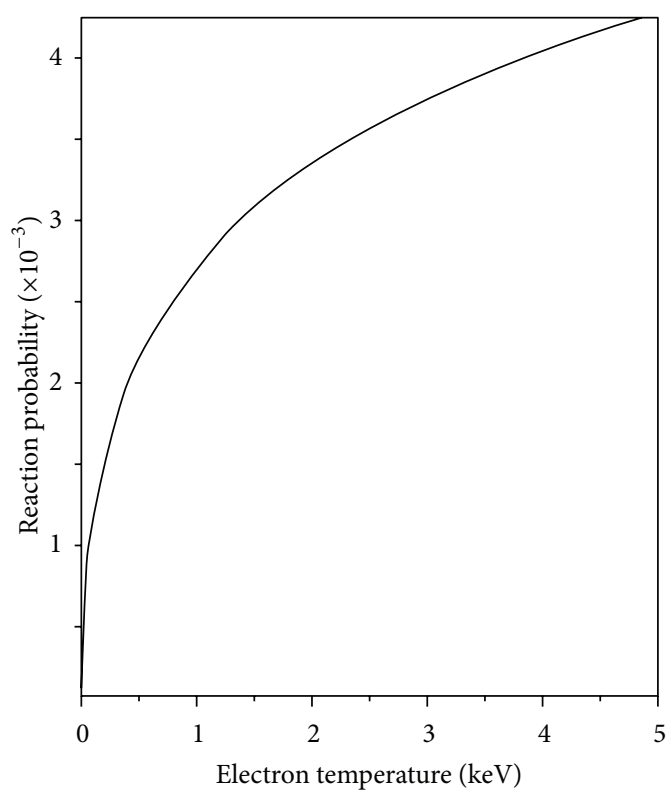

FIGURE 3: The probability $F_{\mathrm{TD}}\left(E_{\mathrm{th}}\right)$ of the in-flight $\mathrm{D}(\mathrm{t}, \mathrm{n}){ }^{4} \mathrm{He}$ reaction in the $\mathrm{D} /{ }^{3} \mathrm{He}$ plasma induced by the $191 \mathrm{keV}$ tritium in terms of electron temperature. Density $\mathrm{n}_{\mathrm{D}}=\mathrm{n}_{3_{\mathrm{He}}}=10^{21} \mathrm{~cm}^{-3}$ and ion temperature $T_{i}=100 \mathrm{eV}$.

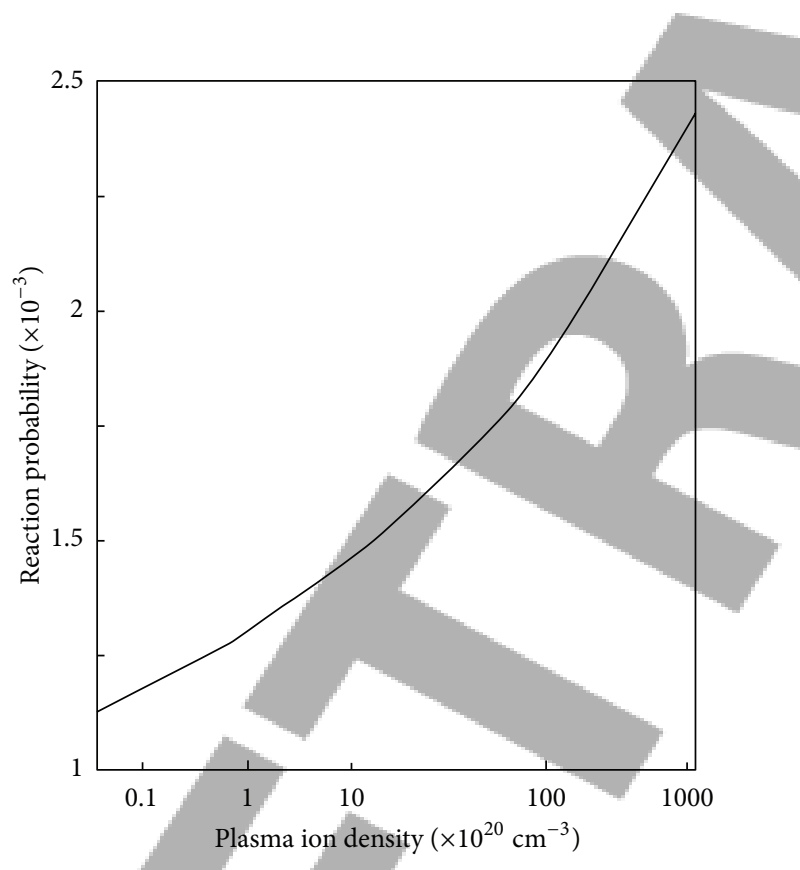

FIgURE 4: The probability $F_{\mathrm{TD}}\left(E_{\mathrm{th}}\right)$ of D + T reaction in the $\mathrm{D} /{ }^{3} \mathrm{He}$ plasma in terms of plasma ion density $\mathrm{n}_{i}=\mathrm{n}_{\mathrm{D}}+\mathrm{n}_{3_{\mathrm{He}}}$. Ion temperature $T_{e}=1 \mathrm{KeV}$ and it is assumed that $\mathrm{n}_{\mathrm{D}}=\mathrm{n}_{3_{\mathrm{H}}}$

relative velocity of the colliding particles in the plasma with the amount of $\bar{\sigma}_{\mathrm{np}} \approx 150 \mathrm{~b}$. By considering the ${ }^{3} \mathrm{He}$ density order of $10^{21} \mathrm{~cm}^{-3}$, the neutron absorption length is $L_{\mathrm{n}} \sim$ $7 \mathrm{~cm}$. Therefore, plasma with 2-3 times the size of neutron absorption length of $(10-20 \mathrm{~cm})$ would be sufficient for the present concept of a fast neutron source. By considering plasma size and the ${ }^{3} \mathrm{He}$ density in the order of $10^{21} \mathrm{~cm}^{-3}$, the neutron absorption probability, $F_{\mathrm{n}^{3} \mathrm{He}}$, is close to unity $\left(F_{\mathrm{n}^{3} \mathrm{He}} \sim 1\right)$. Therefore, $\xi=F_{\mathrm{TD}}\left(E_{\mathrm{th}}\right)$, at electron temperature, $T_{e}=1 \mathrm{KeV}$, the nuclear conversion efficiency, $\xi$, is obtained $2 \times 10^{-3}$. This should be sufficient for a well-yield flux of fast neutrons. For instance, if the flux of thermal neutrons by order $10^{12}-10^{15}\left(\mathrm{n} / \mathrm{cm}^{2} \mathrm{~s}\right)$ as modern neutron sources [9] is injected to plasma, finally, this can lead to the production of $14.1 \mathrm{MeV}$ neutrons by order $10^{9}-10^{12}\left(\mathrm{n} / \mathrm{cm}^{2} \mathrm{~s}\right)$. In this paper, the production of fast neutrons via thermal neutrons is investigated. It is shown that by designing the plasma dimensions, the neutrons are absorbed to helium nuclei at about 90 percent when thermal neutrons enter the considered plasma. By considering the changes of the reaction probability of tritium ions and deuteron in the $\mathrm{D} /{ }^{3} \mathrm{He}$ plasma with respect to electron temperature and ion density, it can be seen that, with the injection of thermal neutron fluxes into the designed $\mathrm{D} /{ }^{3} \mathrm{He}$ plasma in the electron temperature of $T_{e}=1 \mathrm{KeV}$, a fast neutron flux with a nuclear conversion efficiency of $2 \times 10^{-3}$ will be resulted. Therefore it will be enough for the fast neutron flux efficiency.

\section{References}

[1] J. Yu and G. Yu, "Fission-fusion neutron source," Journal of Nuclear Materials C, vol. 386-388, pp. 949-953, 2009.

[2] T. Nakagawa, H. Kawasaki, and K. Shibata, "Curves and tables of neutron cross section in JENDL-3.3 (Part I and II)," JAERIData/Code 2002-02, 2002.

[3] S. B. Borzakov, H. Malecki, L. B. Pikelner, E. I. Sharapov, and M. Stepinski, Soviet Journal of Nuclear Physics, vol. 35, p. 307, 1982.

[4] H. S. Bosch and G. M. Hale, "Improved formulas for fusion cross-sections and thermal reactivities," Nuclear Fusion, vol. 32, no. 4, pp. 611-631, 1992.

[5] D. V. Sivukhin, Reviews of Plasma Physics, vol. 4, Springer, New York, NY, USA, 1963.

[6] S. T. Bulter and M. J. Bucking ham, "Energy loss of a fast ion in a plasma," Physical Review, vol. 126, no. 1, pp. 1-4, 1962.

[7] H. Matsuuraa and Y. Nakao, "Effect of nuclear elastic scattering on ion heating characteristics in deuterium-tritium thermonuclear plasmas," Physics of Plasmas, vol. 13, no. 6, Article ID 062507, 2006.

[8] E. Greenspan and D. Shvarts, "Multigroup model for the slowing-down of energetic ions in plasmas," Nuclear Fusion, vol. 16, no. 2, pp. 295-302, 1976.

[9] V. I. Kukulin, V. T. Voronchev, and B. M. Kuzhevsky, "On neutron generation in beryllium plasma under the effect of a fast-electron beam," Physics of Atomic Nuclei, vol. 68, no. 2, pp. 348-350, 2005. 